\title{
Controladores inteligentes para invernadero hidropónico
}

\author{
Pedro Ponce, Arturo Molina, Omar Mata \\ Tecnológico de Monterrey, Ciudad de México, \\ México \\ pedro.ponce@itesm.mx, armolina@itesm.mx,omar.mata@itesm.mx
}

\begin{abstract}
Resumen. En este caso de estudio se presenta el diseño de un prototipo funcional de un invernadero hidropónico inteligente que permite cultivar frutos, como el jitomate, durante cualquier época del año, manipulando variables específicas como la temperatura y la humedad del microclima dentro del invernadero. Los objetivos del presente caso estudio son analizar las partes que componen el prototipo como es la estructura del invernadero. Se analiza de manera particular el efecto que el viento de la zona provoca sobre esta, así como describir e implementar diferentes técnicas de control básico e inteligente para manipular el microclima en el invernadero. La metodología a seguir inicia por describir las características del cultivo, las condiciones climáticas de la zona, ubicación del invernadero, orientación, material de la estructura, la cubierta y la cimentación, además de implementar dispositivos para la automatización, técnicas de control y herramientas de simulación como Matlab ${ }^{\circledR}$ y LabVIEW ${ }^{\mathrm{TM}}$, que se aplican para la generación y manipulación del microclima. Los resultados muestran características mecánicas como los desplazamientos, los esfuerzos, las tensiones, los momentos o las fuerzas de reacción de los invernaderos con estructuras tipo túnel con cenital, mariposa, diente de sierra, multi-túnel, parral capilla y sombrero chino, para determinar la más factible a ser construida respecto al clima de la Ciudad de México. También se describe la simulación del comportamiento estructural y del sistema de control inteligente del invernadero que se construyó en el Instituto Tecnológico y de Estudios Superiores de Monterrey campus Ciudad de México (ITESM CCM).
\end{abstract}

Palabras clave: invernadero, estructuras, sistemas de control, materiales, clima.

\section{Smart Hydroponic Greenhouse Controllers}

\begin{abstract}
In this case study we present the design of a functional prototype of an intelligent hydroponic greenhouse that allows to grow fruits, like tomato, at any time of the year, manipulating specific variables such as temperature and humidity of the microclimate inside the greenhouse. The objectives of the present case study are to analyze the parts that compose the prototype as it is the structure of the greenhouse.
\end{abstract}


The effect of the wind in the region over the structure is analyzed. And to describe and implement different basic control techniques and intelligent techniques to manipulate the microclimate in the greenhouse. The methodology to be followed begins by describing the characteristics of the crop, the climatic conditions of the area, the location of the greenhouse, its orientation and structure material, its cover and foundation, as well as implementing devices for automation, control techniques and simulation tools such as Matlab ${ }^{\circledR}$ and LabVIEWTM, which are applied for the generation and manipulation of the microclimate. The results show mechanical characteristics such as displacements, stresses, moments or reaction forces of greenhouses with tunnel structures with zenith, butterfly, sawtooth, multi-tunnel, chapel and Chinese hat, to determine the most feasible to be built with respect to the climate of Mexico City. Simulation of the structural behavior and the intelligent control system of the greenhouse is also described, which was built at the Instituto Tecnologico y de Estudios Superiores de Monterrey campus in Mexico City (ITESM CCM).

Keywords: greenhouse, structures, control systems, materials, climate.

\section{Introducción}

En México, se realizan inversiones privadas y públicas para la instalación, operación y automatización de estructuras para tener agricultura protegida (AP) como invernaderos, macrotúneles, malla anti-insectos o casa sombra [1]. Las hectáreas protegidas han evolucionado desde 1998 a 2008 a una Tasa Media de Crecimiento Anual (TMCA) de $34.5 \%$. Moreno et al. [2] mencionan que en 2008 de 8,834 ha con AP, 49\% eran de invernaderos tecnificados y de mediana tecnificación y el $5 \%$ de malla sombra. Si este tipo de invernaderos considera el uso de la hidroponía para obtener mayores rendimientos que por unidad de superficie tienen de 100 a $3000 \%$ más que el cultivo convencional en el suelo [3]. La tasa de crecimiento nacional de la agricultura protegida es de $20 \%$, aunque los proyectos instrumentados y controlados en el sector presentan tasas de sobrevivencia de $60 \%$ [4], debido a la falta de tecnologías adecuadas para las condiciones particulares de cada región del país y a la instalación de estructuras en lugares inapropiados que incrementan los costos de inversión y de producción. Para aumentar la producción de un cultivo es necesario optimizar la tecnología aplicada con técnicas de control específicas para manipular el microclima dentro de un invernadero. Desde 1970 se usan técnicas de control en los invernaderos como el PID (control proporcional + integral + derivativo) o el control apagado/encendido que son técnicas convencionales y los controles óptimo y predictivo, que son técnicas avanzadas, aplicando modelos del clima por ser no lineal e inestable. El principal objetivo del modelado del microclima es tener una primera aproximación del comportamiento del sistema antes de validar e implementar en un invernadero real. Lafont y Balmat [5] explican el uso de la teoría del control difuso para identificar el sistema del invernadero basado en datos de entrada y salida. Bennis et 
al. [6] mencionan otras técnicas como: el modelo predictivo, predictivo generalizado, óptimo, lineal cuadrático adaptivo, redes neuronales, lógica difusa, no lineal, control robusto o el control compatible. Las técnicas de control convencional son importantes en las aplicaciones reales de ingeniería, pero generalmente no todas consideran las propiedades como la interacción entre las variables, las no linealidades, las múltiples restricciones y los conflictos entre objetivos que pueden existir en el sistema de control del microclima del invernadero, siendo necesario hacer una combinación entre ellas como menciona Haigen $\mathrm{Hu}$ [7].

\subsection{Técnicas de control para manipulación del microclima}

Tabla 1. Características básicas de controladores empleados en invernaderos.

\begin{tabular}{lllll}
\hline Controlador & $\begin{array}{l}\text { Nivel de } \\
\text { tecnología }\end{array}$ & $\begin{array}{l}\text { Complejidad de } \\
\text { implementación }\end{array}$ & $\begin{array}{l}\text { Complejidad de } \\
\text { uso }\end{array}$ & Mantenimiento \\
\hline CTDSR & Bajo & Bajo & Bajo & Bajo \\
$\begin{array}{l}\text { Encendido- } \\
\text { apagado }\end{array}$ & Bajo & Bajo & Bajo & Medio \\
PID & Medio & Bajo & Bajo & Bajo \\
Fuzzy & Alto & Medio & Medio & Medio
\end{tabular}

Tabla 2. Tipos de control para invernadero con base en el clima.

\begin{tabular}{lll}
\hline Clima & Tipo de Control & Invernadero \\
\hline \hline $\begin{array}{l}\text { Climas tropicales o desérticos } \\
\text { secos }\end{array}$ & PID, FUZZY & $\begin{array}{l}\text { Túnel con cenital, Mariposa, Multi-Túnel, } \\
\text { Capilla, Sombrero Chino }\end{array}$ \\
\hline \hline $\begin{array}{l}\text { Desierto subtropical y climas } \\
\text { mediterráneos }\end{array}$ & CDTWF, ON- & $\begin{array}{l}\text { Túnel con cenital, Mariposa, Multi-Túnel, } \\
\text { Capilla, Sombrero Chino }\end{array}$ \\
\hline \hline Climas tropicales húmedos & CDTWF, ON- & $\begin{array}{l}\text { Diente de sierra, Parral modular, Capilla, } \\
\text { Túnel con cenital, Mariposa, Sombrero } \\
\text { chino }\end{array}$ \\
& OFF, PID, Fuzzy & $\begin{array}{l}\text { Túnel con cenital, Mariposa, Multi-Túnel, } \\
\text { Capilla }\end{array}$ \\
\hline \hline Climas templados & ON-OFF, PID, & \\
\hline \hline Climas templados fríos en & ON-OFF, PID, & Venlo \\
latitudes superiores a 45 ${ }^{\circ}:$ & FUZZY & \\
\hline
\end{tabular}


El control aplicado a elementos mecánicos, eléctricos y electrónicos de un invernadero, se implementa considerando la estructura física, ventilación, sistema de humidificación, riego y suministro de nutrientes usando sensores, actuadores, un controlador y técnicas de control para regular el microclima en el interior del invernadero. Los tipos de control aplicados a los invernaderos son: control por tiempo definido por la experiencia del agricultor, encendido-apagado y control PID. Respecto a la complejidad, características de instalación y uso de técnicas de control, se muestra en la Tabla 1 un resumen con base en el tipo de control a emplear y en la Tabla 2 se propone el control aplicado a un tipo de clima específico.

Existen zonas específicas (por ejemplo, climas extremos de invierno) o tipos de cultivo como las rosas que requieren de muchos cuidados y control de las variables, demostrando que este tipo de control no es suficiente, siendo necesario tener un sistema de control inteligente.

\subsection{Estrategias de control inteligente}

Existen invernaderos que utilizan el control inteligente para la manipulación del microclima, así como para el suministro de nutrientes del cultivo. Para diseñar el control se requiere un lazo cerrado (Figura 1), que involucra sensores, actuadores y estrategias de control que son incluidos en el modelo dinámico del invernadero. Para resolver la función de transferencia de este modelo, los métodos no lineales que se emplearon en este caso de estudio son los expuestos por Isidori, Sastry y Khalil $[8,9,10]$.

Realimentación/ prealimentación linealizada: $\mathrm{Su}$ objetivo es transformar algebraicamente un sistema dinámico no lineal en uno lineal que otras técnicas de control lineal pueden aplicar.

Control de modo deslizamiento (SMC): La acción del controlador es conmutar a una frecuencia específica. La regla de control es usada para manipular el error del sistema a un híper plano en el espacio de estados, cuando la superficie de deslizamiento es alcanzada, el estado del sistema se mantiene en conmutación o deslizamiento hasta tener un estado de equilibrio.

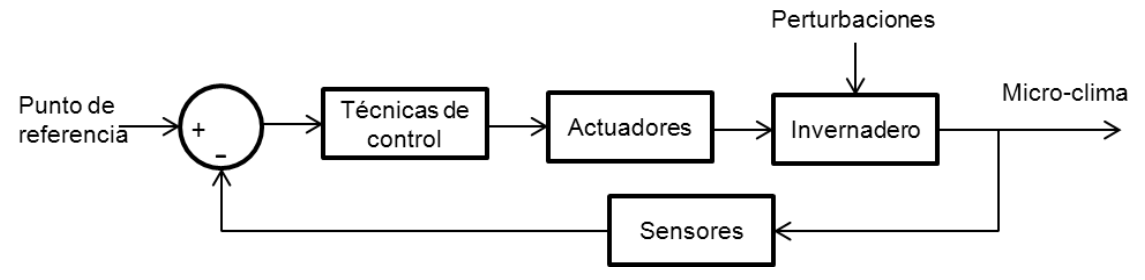

Fig. 1. Esquema de lazo de control de un invernadero.

Lógica difusa: Su objetivo es construir un modelo capaz de controlar la planta del sistema sin usar modelos en términos matemáticos. Los sistemas de lógica difusa son muy 
utilizados en dos contextos generales: en situaciones de sistemas altamente complejos cuyos comportamientos no son muy claros y en situaciones donde se necesita una rápida aproximación.

ANFIS (Sistema de inferencia neuro- difuso adaptivo): Es una representación gráfica de una red con un sistema difuso de tipo T-S. Esta combina los beneficios de una red neuronal con la inferencia de un sistema difuso, aprovechando un mismo modelo que tiene como ventajas ser más rápido aprendiendo, exacto y mejor capacidad de gestión de datos. Tiene un sistema de inferencia difuso, donde los parámetros de la función de membresía se ajustan por la formación de un algoritmo hibrido que combina el gradiente descendiente y el método de mínimos cuadrados.

\section{Estructura del invernadero}

Con base en la mecánica de los materiales y las consideraciones estructurales como la forma o las dimensiones, se realizó la simulación del impacto del viento en invernaderos como túnel con cenital, mariposa diente de sierra, multitunel, parral capilla y sombrero chino en el programa INVENTOR ${ }^{\circledR}$ de AUTODESK $®$, para obtener características mecánicas como los desplazamientos, los esfuerzos, las tensiones, los momentos o las fuerzas de reacción y seleccionar el que presente menos problemas estructurales. Por la magnitud de información que se obtiene en el proceso INVENTOR ${ }^{\circledR}$, solo se reportan los invernaderos que tienen valores más altos en las variables mostradas en la Tabla 3, teniendo como condiciones iniciales: velocidad de viento de $30 \mathrm{~km} / \mathrm{hr}$ (como lo indico el Servicio Meteorológico Nacional en otoño del 2013, que puede consultarse en CONAGUA [11]), orientación Norte-Sur y material de la estructura en acero galvanizado. En la Figura 2 se muestra un ejemplo del análisis realizado a un invernadero tipo Venlo.

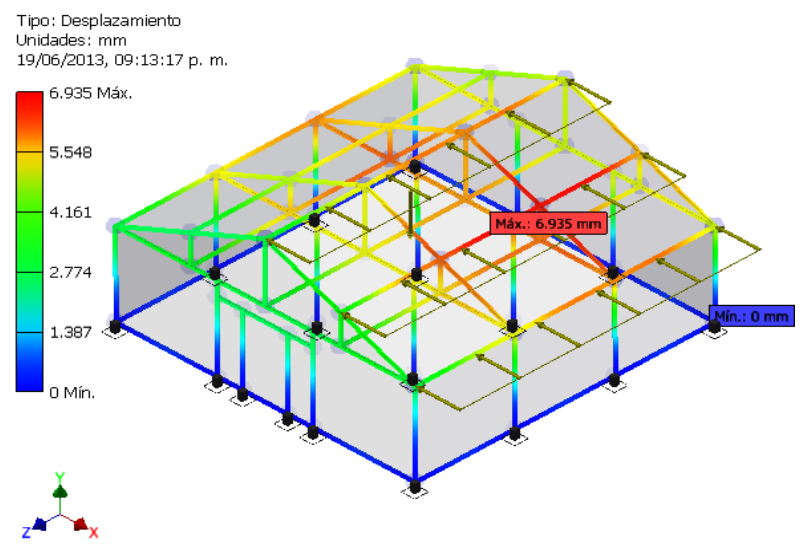

Fig. 2. Análisis mecánico de un invernadero tipo venlo. 
Tabla 3. Características mecánicas de diferentes invernaderos después de inducir una fuerza a través de viento sobre la estructura.

\begin{tabular}{lllllll}
\hline $\begin{array}{l}\text { Características } \\
\text { / tipo de } \\
\text { invernadero }\end{array}$ & $\begin{array}{l}\text { Desplazamiento } \\
(\mathrm{mm})\end{array}$ & $\begin{array}{l}\text { Fuerza } \\
\text { de } \\
\text { reacción } \\
(\mathrm{N})\end{array}$ & $\begin{array}{l}\text { Momento } \\
(\mathrm{Nmm})\end{array}$ & $\begin{array}{l}\text { Esfuerzo } \\
\text { normal } \\
(\mathrm{MPa})\end{array}$ & $\begin{array}{l}\text { Tensión } \\
\text { de corte } \\
(\mathrm{MPa})\end{array}$ & $\begin{array}{l}\text { Esfuerzo } \\
\text { de } \\
\text { torsión } \\
(\mathrm{MPa})\end{array}$ \\
\hline \hline $\begin{array}{l}\text { Túnel con } \\
\text { cenital }\end{array}$ & 4.876 & 155.762 & 68379.112 & 12.792 & 0.499 & 0.855 \\
\hline Mariposa & 45.908 & 229.480 & 456595.175 & 85.416 & 1.599 & 2.109 \\
\hline \hline $\begin{array}{l}\text { Diente de } \\
\text { Sierra }\end{array}$ & 10.824 & 394.935 & 271493.092 & 50.789 & 1.549 & 0.838 \\
\hline Multi- túnel & 4.888 & 327.602 & 194233.670 & 46.866 & 1.095 & 0.388 \\
\hline Parral & 7.011 & 386.000 & 141205.529 & 29.003 & 2.156 & 2.170 \\
\hline Túnel & 6.242 & 67.523 & 91531.392 & 17.619 & 0.275 & 0.439 \\
\hline \hline Capilla & 6.935 & 304.677 & 185057.571 & 34.619 & 1.193 & 0.636 \\
\hline $\begin{array}{l}\text { Sombrero } \\
\text { Chino }\end{array}$ & 4.535 & 318.104 & 126381.397 & 23.959 & 1.343 & 0.583 \\
\hline
\end{tabular}

Como se observa los invernaderos que tienen la base tipo túnel presentan un menor desplazamiento y movimiento en los nodos de las estructuras. Respecto a la fuerza de reacción la que más soporta es el diente de sierra. Esta información también sirve para determinar que invernaderos pueden construirse en batería o grupo por tener una excelente ventilación, tales como: Túnel con Cenital, Mariposa, Diente de Sierra, MultiTúnel y Sombrero Chino. Los invernaderos tipo Túnel ofrecen ventajas que permiten modificaciones como ventanas cenitales o techos tipo sombrero chino o mariposa para mejor ventilación e intercambio de calor

\subsection{Implementación de las técnicas de control}

Las combinaciones de los sistemas de control para hacer un sistema inteligente que se implementan en el invernadero hidropónico son:

Modos deslizantes + Control de linealización de realimentación/prealimentación: Es un modelo espacio estado con acoplamiento no-lineal para el clima del invernadero, las ecuaciones usadas en el sistema son 1 y 2, el lazo de control correspondiente se presenta en la Figura 3:

$$
\begin{gathered}
\mathrm{u}_{1}=\frac{(1+\propto) \mathrm{v}_{1}-\delta \beta_{\mathrm{T}} \mathrm{x}_{2}+\mathrm{UA}\left(\mathrm{v}_{2}-\mathrm{x}_{1}\right)-\rho \mathrm{C}_{\mathrm{p}} \mathrm{V}_{\widehat{\mathrm{u}}_{1}}-\delta \rho \mathrm{V}_{\widehat{\mathrm{u}}_{2}}}{\delta \rho\left(\mathrm{x}_{2}-\mathrm{v}_{3}\right)+\rho \mathrm{C}_{\mathrm{p}}\left(\mathrm{x}_{1}-\mathrm{v}_{2}\right)}, \\
\mathrm{u}_{2}=\frac{\rho \mathrm{C}_{\mathrm{p}}\left(\mathrm{x}_{1}-\mathrm{v}_{2}\right)\left(-\frac{\alpha}{\delta} \mathrm{v}_{1}+\delta_{\mathrm{Tx}_{2}}+\rho \mathrm{V}_{\widehat{\mathrm{u}}_{2}}\right)+\rho\left(\mathrm{x}_{2}-\mathrm{v}_{3}\right)\left(\mathrm{v}_{1}+U A\left(\mathrm{v}_{2}-\mathrm{x}_{1}\right)-\rho \mathrm{C}_{\mathrm{p}} \mathrm{V}_{\widehat{\mathrm{u}}_{1}}\right)}{\delta \rho\left(\mathrm{x}_{2}-\mathrm{v}_{3}\right)+\rho \mathrm{C}_{\mathrm{p}}\left(\mathrm{x}_{1}-\mathrm{v}_{2}\right)},
\end{gathered}
$$


dónde $\mathrm{C}_{\mathrm{p}}$ es calor especifico del aire, $\rho$ densidad del aire, $\mathrm{V}$ es volumen de aire, $\propto$ es coeficiente global para usar la sombra y las dimensiones del área, $\beta_{\mathrm{T}}$ es coeficiente global de las constantes termodinámicas, $\mathrm{x}_{1}$ y $\mathrm{x}_{2}$ son variables de estado dinámico (temperatura y humedad absoluta), $\mathbf{u}_{1}$ y $\mathbf{u}_{2}$ es rango de ventilación y capacidad de humedad del sistema de niebla:

$$
\begin{aligned}
& \hat{\mathrm{u}}_{1}=\mathrm{k}_{1} \operatorname{sgn}\left(\mathrm{x}_{1}-\mathrm{x}_{\mathrm{d}_{1}}\right), \\
& \hat{\mathrm{u}}_{2}=\mathrm{k}_{2} \operatorname{sgn}\left(\mathrm{x}_{2}-\mathrm{x}_{\mathrm{d}_{2}}\right) .
\end{aligned}
$$

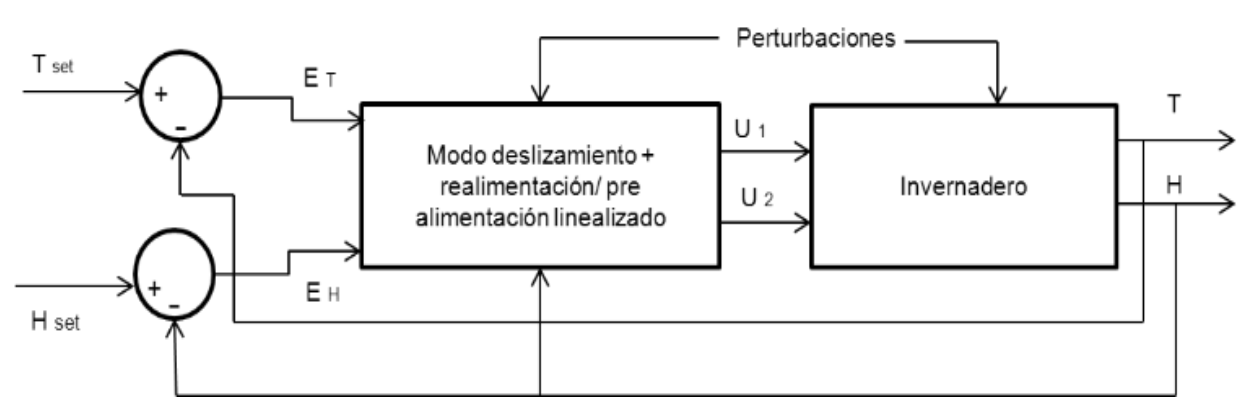

Fig. 3. Lazo de control de clima.

Control de lógica difusa + ANFIS: Este se aplica al sistema de irrigación y al de hidroponía, para su uso se implementa el control en un tanque que debe ser monitoreado y controlado. La importancia de este radica en la dificultad y complejidad del sistema para obtener los datos experimentales. El modelo matemático para la alimentación de nutrientes es derivado por un balance de masas en los tanques de alimentación y colectores, además de un balance de materia para la concentración de los nutrientes en el tanque colector (ecuaciones de la 5 a la 8). La propuesta de control para alimentación de nutrientes se basa en la lógica difusa, cuyo modelo se muestra en la Figura 4, utilizando también ANFIS. El controlador consiste en tres entradas: altura del tanque de alimentación $\left(\mathrm{h}_{1}\right)$, altura del tanque colector $\left(\mathrm{h}_{2}\right)$, el error de conductividad eléctrica de los sensores $\left(\mathrm{EC}_{\text {error }}\right)$ y una salida que es el tiempo $(\mathrm{t})$ de activación del solenoide; las ecuaciones para el modelo son:

Ecuación de la válvula:

$$
f(t)=C_{v} \sqrt{\frac{\mathrm{ph}_{1}(t)}{144}}
$$

Tanque de alimentación, balance de masa:

$$
\frac{d}{d t} h_{1}(t)=-\frac{f(t)}{A_{1}} \text {. }
$$


Pedro Ponce, Arturo Molina, Omar Mata

Tanque colector, balance de masa:

$$
\frac{\mathrm{d}}{\mathrm{dt}} \mathrm{h}_{2}(\mathrm{t})=-\frac{\mathrm{f}(\mathrm{t})}{\mathrm{A}_{2}}
$$

Concentración (ppm) o conductividad eléctrica $(\mathrm{mS} / \mathrm{cm})$ :

$\mathrm{EC}=0.0014 \mathrm{C}+0.0318$

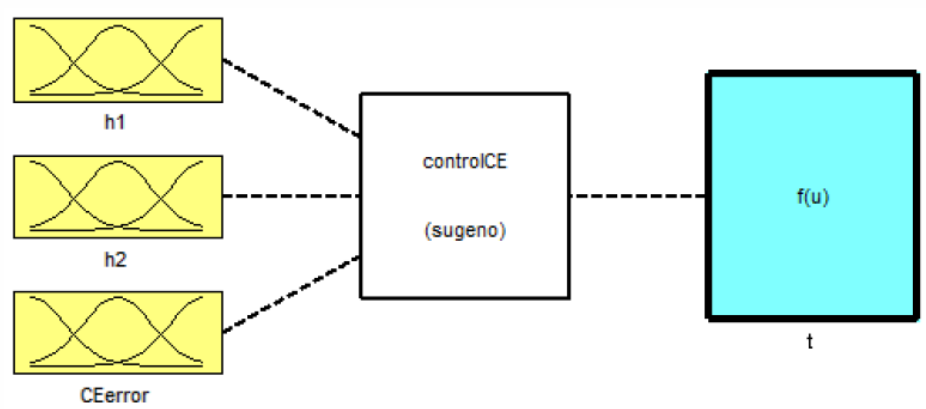

Fig. 4. Modelo logica difusa para alimentación de nutrientes.

Se tiene una configuración de un sistema ANFIS, como se muestra en la Figura 5. Esta presenta cada entrada y consiste en 3 funciones de membresía (Figura 6) y 27 reglas diferentes que se basan en la altura de los tanques y el error en la conductividad eléctrica.

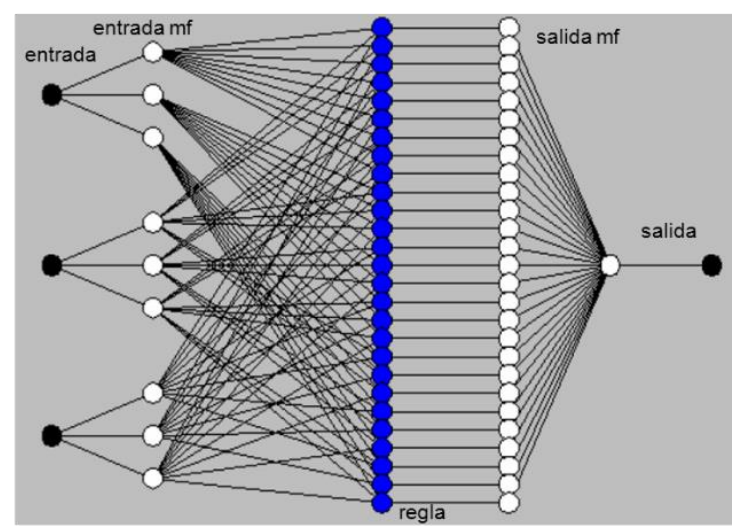

Fig. 5. Configuración de un sistema ANFIS. 

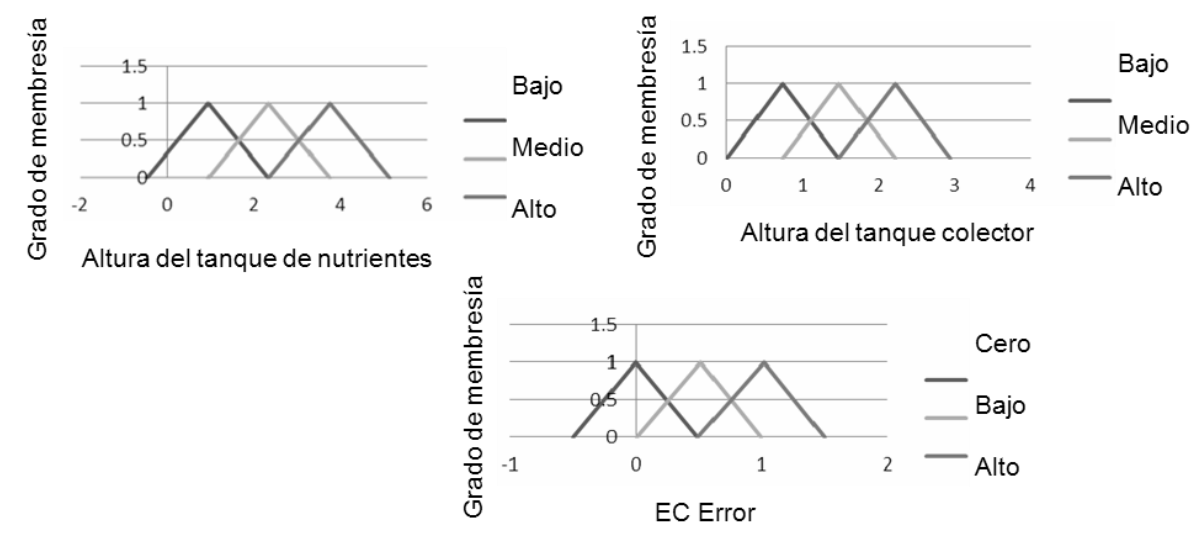

Fig. 6. Funciones de membresia.

La salida t consiste de 27 funciones linelaes de primer orden polinomial de la forma:

$$
\mathrm{t}=\left(\mathrm{a} * \mathrm{~h}_{1}\right)+\left(\mathrm{b} * \mathrm{~h}_{2}\right)+\left(\mathrm{c} * \mathrm{EC}_{\text {error }}\right)+\mathrm{d},
$$

donde a,b,c y d son constantes.

Finalmente el lazo de control de alimentacion de los nutrientes es el siguiente (Figura 7):

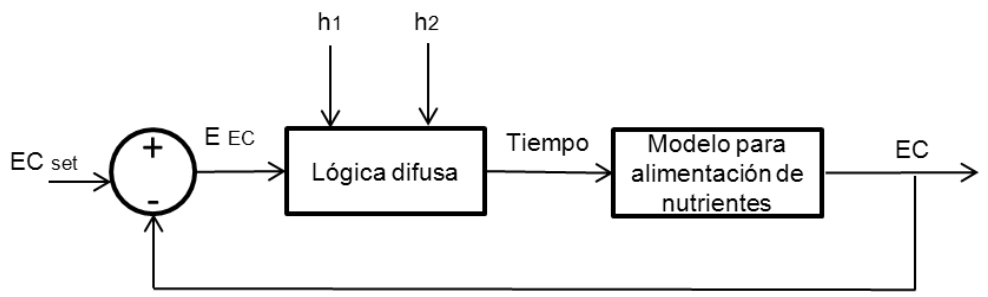

Fig. 7. Lazo de control para alimentación de nutrientes.

Los controladores administran diferentes actuadores para lograr un buen funcionamiento en términos del microclima deseado para el cultivo del jitomate, estos se activan o no dependiendo de la adquisición de señales que indique si las variables se encuentran o no dentro del rango preestablecido. La simulación del microclima se hizo con LabVIEW ${ }^{\text {TM }} 2012$ y dos herramientas auxiliares: el ICTL (por sus siglas en ingles Intelligent Control Toolkit LabVIEW ${ }^{\mathrm{TM}}$ ) y un simulador del diseño de control como MATLAB $®$, para obtener gráficas con condiciones distintas de temperatura y humedad relativa que permiten determinar el tipo de control que estabiliza más rápido el sistema a situaciones con cambios repentinos en el clima. En la Figura 8 se muestra el control 
convencional de humedad relativa y control de temperatura que se activan o no dependiendo de las variables medidas, si están o no fuera del rango preestablecido.

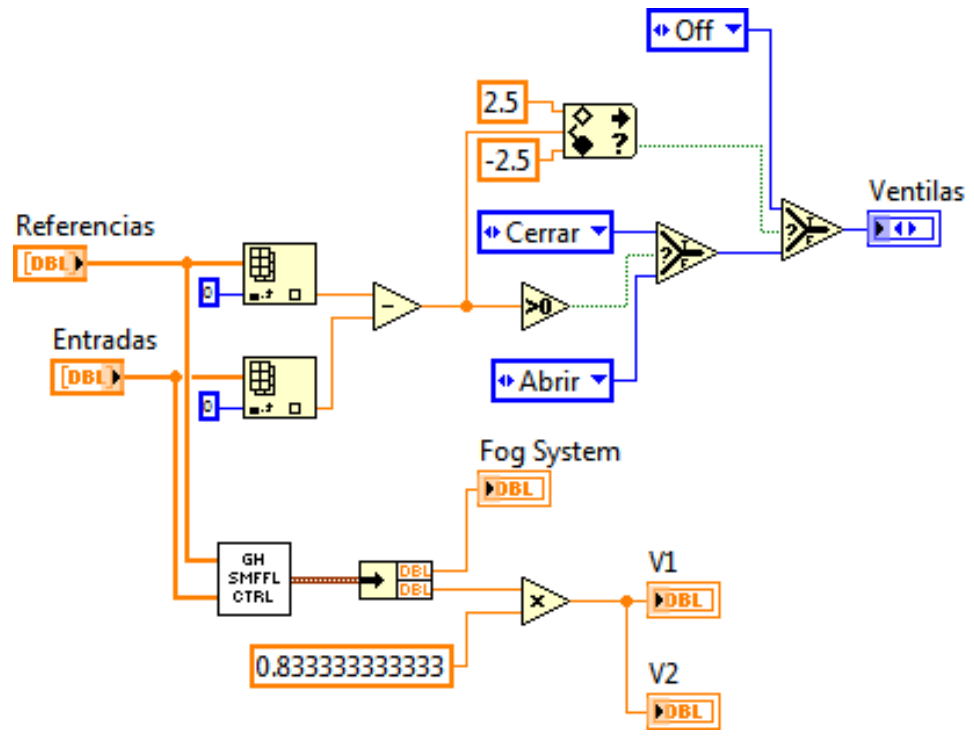

Fig. 8. Control no lineal de temperatura y de humedad relativa en el programa LabVIEW TM.

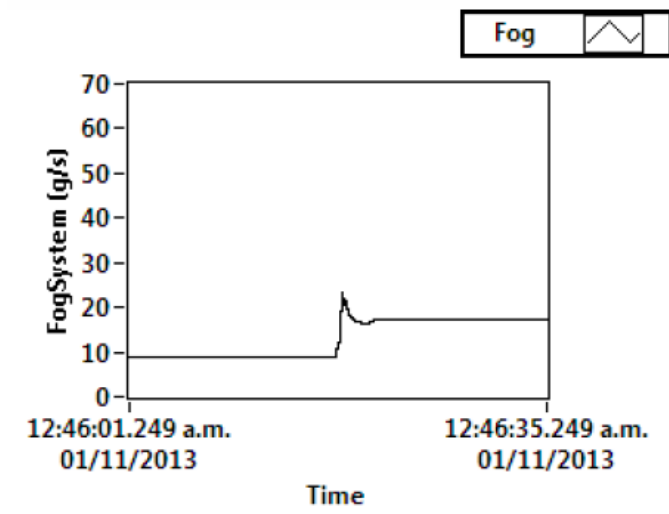

Fig. 9. Respuesta del sistema de nebulización (Fogsystem) ante perturbaciones aplicando un control de Modo deslizamiento + Control de Linealización de realimentación /prealimentación.

Se eligieron como base de simulación los valores deseados para el cultivo del tomate. Las condiciones dentro del invernadero fueron de $30{ }^{\circ} \mathrm{C}$ y $20 \%$ de humedad relativa 
respectivamente. La intensidad de luz fue de $300 \mathrm{w} / \mathrm{m} 2$, la temperatura fuera del invernadero es de $27{ }^{\circ} \mathrm{C}$ y $15 \%$ de humedad relativa. Algunos cambios y manipulaciones de variables que se hicieron en los puntos deseados para realizar pruebas fueron de $30{ }^{\circ} \mathrm{C}$ a $23{ }^{\circ} \mathrm{C}$ en la temperatura y del $20 \%$ al $60 \%$ en la humedad relativa. Se realizaron pruebas aplicando los controles referidos anteriormente obteniéndose que el mejor es el Modo deslizamiento + Control de Linealización de realimentación /prealimentación; porque tiene una respuesta suave para alcanzar el punto de ajuste. Éste puede ser un poco más bajo que el PID, pero el segundo es menos estable y se ve como oscilaciones en la parte del sistema de nebulización como se muestra en las Figuras 9 y 10.

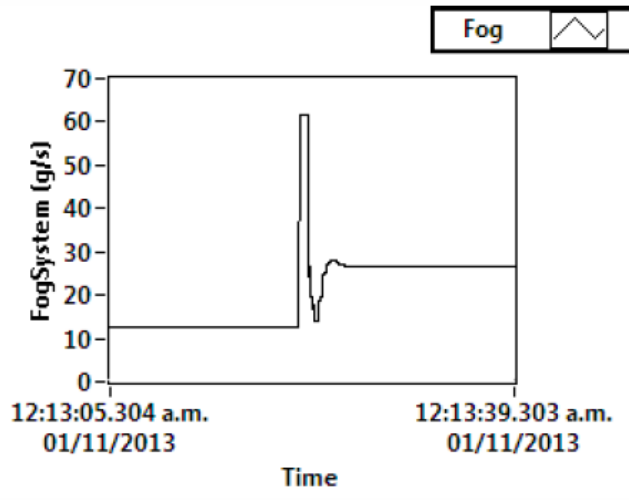

Fig. 10. Respuesta del sistema de nebulización ante perturbaciones aplicando un control PID.

\subsection{Interfaz gráfica de usuario}

El principal objetivo de la interfaz gráfica de usuario (IGU) es dar la posibilidad de programar las necesidades del cultivo en términos del microclima, de las etapas de crecimiento y de los nutrientes que se requieran, lo que implica que la IGU automáticamente resolverá las demandas del cultivo programadas por el usuario (Figura 11).

\subsection{Implementación de invernadero hidropónico inteligente}

El invernadero que se tiene en el ITESM CCM (Figura 12), tiene una orientación Norte-Sur en un clima templado subhúmedo con vientos que circulan a una velocidad aproximada de $30 \mathrm{~km} / \mathrm{h}$ en otoño. La zona en que se instaló está rodeada de árboles que sirven como protección contra el viento; cuenta con servicios para un buen funcionamiento como: agua, electricidad e internet para la transmisión de datos en tiempo real principalmente. 
INVERNADERO INTELIGENTE TECNOLÓGICO DE MONTERREY
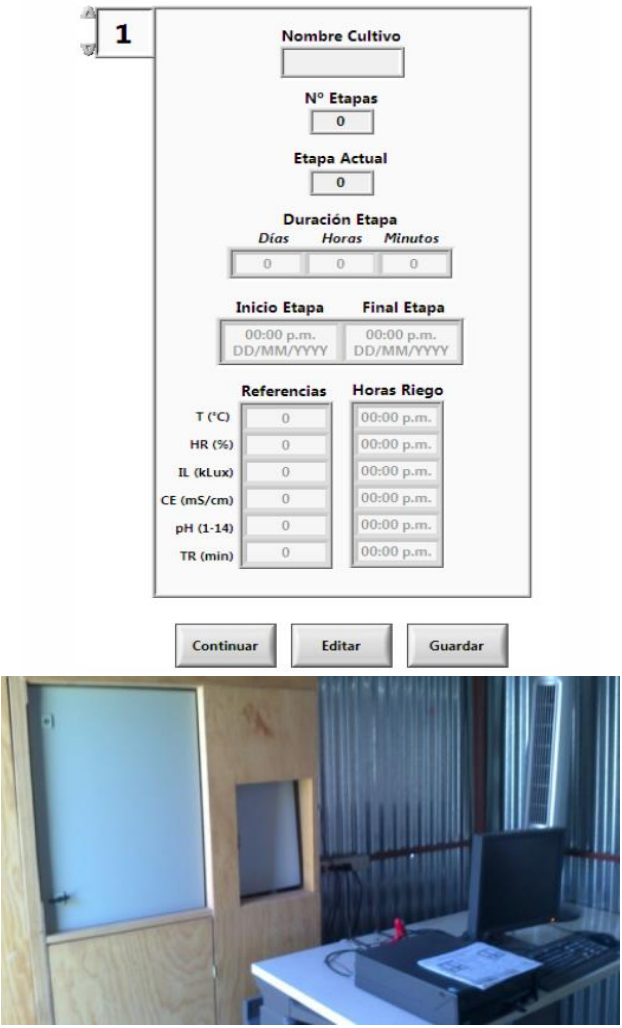

Fig. 11. Panel de control y caseta de control.

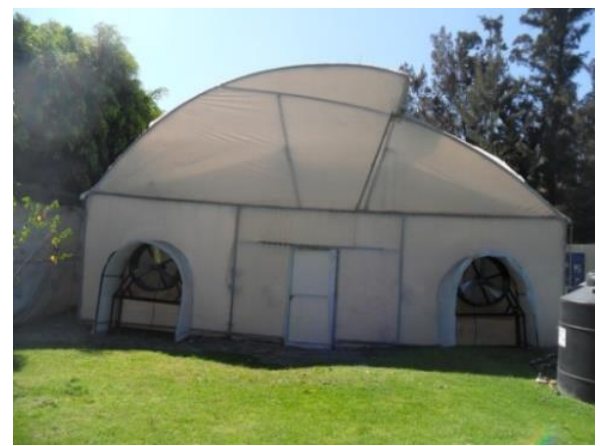

Fig. 12. Invernadero inteligente. 
La estructura tipo semi-túnel, cuenta con una ventana cenital y dos laterales para prevenir acumulación de agua y mejorar la circulación del viento, además de una regulación de la temperatura. Tiene un área de cultivo de $100 \mathrm{~m} 2$. La estructura permite resistir vientos con velocidades de $75 \mathrm{~km} / \mathrm{hrs}$ y soportar cargas de $25 \mathrm{~kg} / \mathrm{m} 2$; tiene soportes para el tutoreo de las plantas. El diseño de la estructura es de tipo modular y utiliza columnas, conectores, largueros y arcos galvanizados con perfil tubular de 1.5 pulgadas. Esto se seleccionó por los resultados del análisis de esfuerzos en INVENTOR ${ }^{\circledR}$, obteniéndose que la tensión más alta de los invernaderos es del $1 \%$ (2.156 Mpa) de la tensión máxima admisible, haciendo que el acero galvanizado sea un excelente material para la estructura. La cubierta del invernadero es de plástico tratado con estabilizador de rayos UV II calibre 720.

Se implementaron equipos que permiten la extracción del aire. En la Figura 13 se presenta la simulación de viento en el programa CFD de Inventor ${ }^{\circledR}$ dentro y fuera del invernadero. Esta se realizó en un túnel de viento con el propósito de determinar qué tan eficaz es la construcción teniendo las siguientes características: estructura del material acero galvanizado, material de la cubierta es polietileno de baja densidad, velocidad del viento de $30 \mathrm{~km} / \mathrm{hrs}$, viento tipo Barlovento, dimensiones del túnel $7 \mathrm{~m}$ de alto, $14 \mathrm{~m} \mathrm{de}$ profundidad y $20 \mathrm{~m}$ de ancho y una temperatura de $19.85^{\circ} \mathrm{C}$.

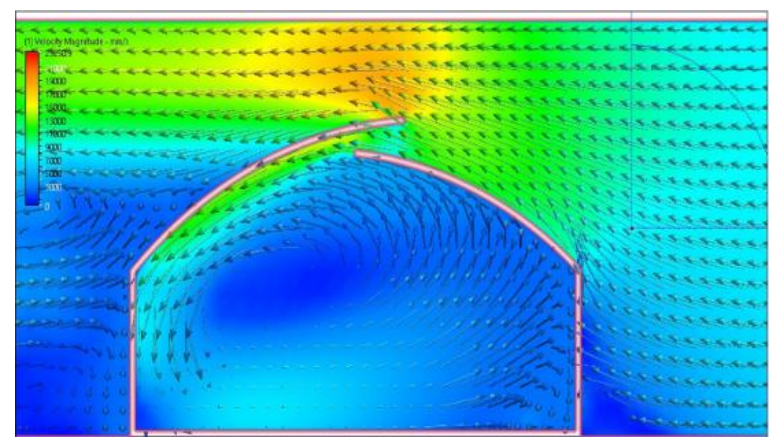

Fig. 13. Simulación del comportamiento del viento en CFD de INVENTOR ®.

Debe considerarse que, para mejorar los resultados de la construcción, es necesario hacer simulaciones con los cambios de velocidad del viento, dirección del invernadero, tipo de cultivo y diferentes tipos de materiales. En el interior del invernadero se cuenta con un sistema de sombreo, que consiste en una malla que protege el cultivo contra radiación solar, permitiendo la regulación de la intensidad de luz. Se instaló para la protección del cultivo en una extensión de $100 \mathrm{~m} 2$, y tiene actuadores independientes tanto en la parte superior como en la inferior, del lado izquierdo y derecho para su mejor manipulación. Se instaló un sistema de humidificación que utiliza un sistema de nebulización para ajustar la humedad relativa y para controlar la regulación de temperatura y un sistema de riego que se basa en una técnica hidropónica llamada NFT 
(Técnica de la Película Nutriente) que consiste en recircular una solución de nutrientes a través de un sistema de tuberías alimentando el cultivo, junto con éste se coloca un sistema de suministro de nutrientes, que consiste en tres tanques que almacenan soluciones nutritivas para cada etapa de crecimiento del cultivo. Para controlar el flujo de nutrientes y del agua del sistema de humidificación, se utilizan electroválvulas de 3 vías 2 posiciones de $120 \mathrm{~V} \mathrm{50/60} \mathrm{Hz} \mathrm{y} \mathrm{una} \mathrm{bomba} \mathrm{que} \mathrm{se} \mathrm{controla} \mathrm{por} \mathrm{encendido/apagado.} \mathrm{El}$ sistema de control del invernadero inteligente hace la adquisición de datos por medio de sensores para detección de temperatura, humedad relativa, intensidad de luz, conductividad eléctrica, $\mathrm{pH}$ de la solución nutritiva y el nivel de la solución en los tanques colectores y de almacenamiento a través de una tarjeta de adquisición de datos de National Instrument ${ }^{\circledR}$. La localización de los sensores dentro del invernadero se muestra en la Figura 14. Para el uso y aplicación de todos los dispositivos y sensores antes referidos, se hizo un sistema de control, que tiene las siguientes características:

Computadora: Toma las decisiones pre-programadas. Esta es IBM Thinkpad con Windows 7 Professional Edition, procesador Pentium IV a $2.8 \mathrm{GHz}$ y 2 GB de RAM. Tiene instalado LabVIEW ${ }^{\mathrm{TM}}$, software portador de la interfaz de usuario y controladores del Invernadero.

Interfaz de Hardware: NI CompactDAQ-9174 y NI USB-6009 son usados para adquirir y generar señales. Tiene conectado un módulo de entradas analógicas NI-9221 y tres de relés NI-9481.

Estrategias de Control: Se tienen tres tipos de controladores implementados y programados actualmente: a) Manual - El usuario decide la acción a llevarse a cabo, b) encendido/ apagado Convencional - Control automático basado en comparadores, c) No convencional - Control automático basado en estrategias de control no-lineal e inteligentes, Linealización por Realimentación, Modos Deslizantes y Lógica Difusa.

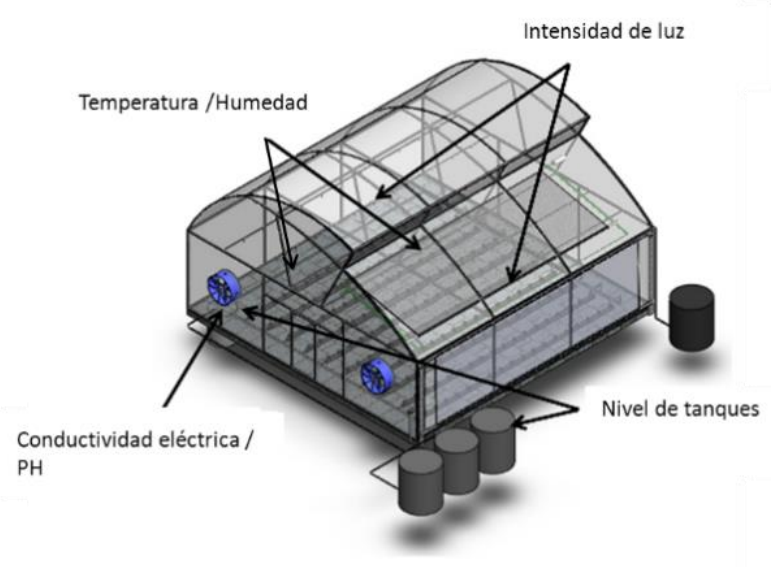

Fig. 14. Localización de los sensores. 


\section{Conclusiones}

Después de la construcción del invernadero, las plantas fueron sembradas el 2 de noviembre del 2013 concluyendo en el mes de mayo del presente año la última recolección del fruto, comprobándose la funcionalidad del sistema de control (Figura 15). En esta fase solo se requirió mantenimiento y limpieza de los aspersores y las válvulas por las que circulan nutrientes. Las actividades que se realizaron por parte del personal encargado solo fueron realizar el tutoreo y la poda de las plantas, ya que se acondiciono un sistema de monitoreo por internet para visualizar algún desperfecto que requiriera atención inmediata.

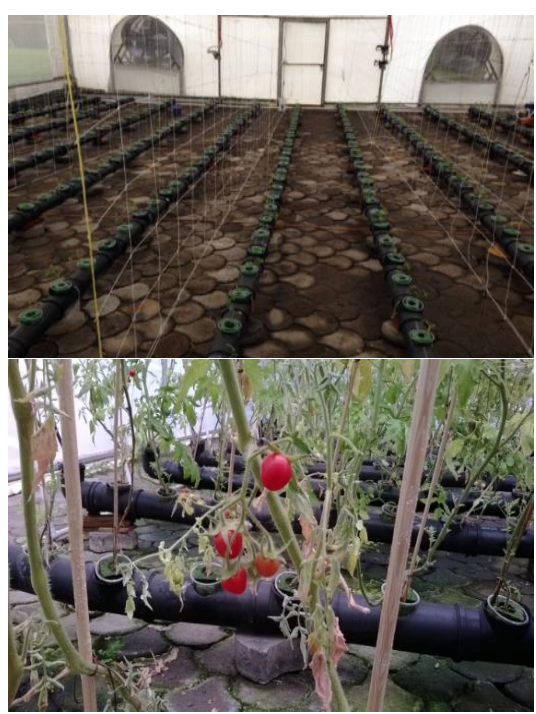

a) Noviembre del 2013

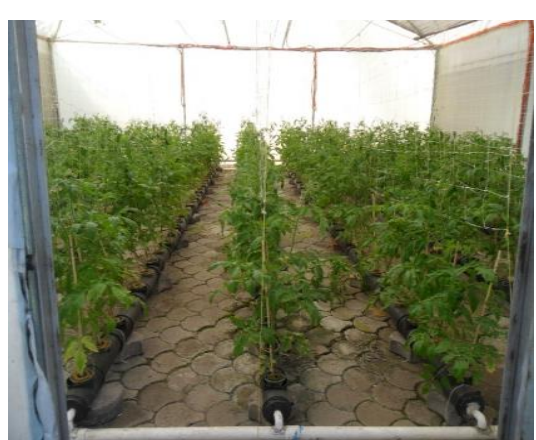

b) Mayo del 2014

Fig. 15. Evolución del cultivo de jitomate del invernadero inteligente del Instituto Tecnológico de Estudios Superiores Monterrey Campus Ciudad de México. 


\section{Referencias}

1. Fideicomisos Instituidos en Relación con la Agricultura.: Oportunidad De Negocios en Agricultura Protegida. Boletín Informativo Fira, pp. 11-98 (2010)

2. Moreno, A., Aguilar, J., Luévano, A.: Características de la Agricultura Protegida y su Entorno en México. Revista Mexicana De Agronegocios, Vol. 15, pp. 763-774 (2011)

3. Jensen, M. H., Collins, W. L.: Hydroponic Vegetable Production. Horticultural Reviews, Vol. 7, pp. 483-558 (1976)

4. Sagarpa: Programa De Ejecución Directa 2009 Agricultura Protegida. Disponible en: Www.Amhpac.Org (2009)

5. Lafont, F., Balmat, J.: Optimized Fuzzy Control of a Greenhouse. Fuzzy Sets and Systems, Vol. 128, pp. 47-59 (2002)

6. Bennis, N., Duplaix, J., Enéa, G., Haloua, M., Youlal, H.: Greenhouse Climate Modelling and Robust Control. Computers and Electronics in Agriculture, pp. 97107 (2008)

7. $\mathrm{Hu}, \mathrm{H} ., \mathrm{Xu}$, L., Wei, R., Zhu, B.: Multi-Objective Control Optimization for Greenhouse Environment Using Evolutionary Algorithms. Sensors, pp. 5792-5807 (2011)

8. Isidori, A.: Nonlinear Control Systems. New York: Springer (1995)

9. Sastry, S.: Nonlinear System: Analysis, Stability, and Control. New York: Springer (1999)

10. Khalil, H. K.: Nonlinear Systems. México: Prentice Hall (2002)

11. Comisión Nacional Del Agua: Servicio Meteorológico Nacional. Disponible en: Http://Smn.Cna.Gob.Mx (2014) 